\title{
Biological significance of localized Type IV scirrhous gastric cancer
}

\author{
KAZUYA ENDO $^{1}$, MASATO SAKURAI $^{1}$, EIJI KUSUMOTO ${ }^{1}$, HIDEO UEHARA ${ }^{1}$, \\ SHOHEI YAMAGUCHI ${ }^{1}$, NORIFUMI TSUTSUMI ${ }^{1}$ and KOJI IKEJIRI ${ }^{1,2}$ \\ ${ }^{1}$ Department of Surgery, Center for Gastroenterology; ${ }^{2}$ Clinical Research Institute, \\ National Kyushu Medical Center, Fukuoka 810-8563, Japan
}

Received June 30, 2011; Accepted October 11, 2011

DOI: $10.3892 / \mathrm{ol} .2011 .454$

\begin{abstract}
The prognosis of type IV scirrhous gastric cancer (SGC) is extremely poor. Linitis plastica (LP), the so-called 'leather bottle stomach', is believed to be a typical case of SGC, which is usually diagnosed as a far-advanced gastric cancer. The pathogenesis of this disease remains unclear. Although typical SGC often invades the entire stomach, atypical cases show SGC localized to one region of the stomach. The aim of the present study was to investigate localized SGC (LSGC) and its biological significance. A total of 509 patients with advanced gastric cancer who underwent gastrectomy were evaluated. These patients were divided into three groups as follows: 19 patients with type IV scirrhous lesions invading the whole stomach (defined as LP), 60 patients with type IV scirrhous lesions localized in less than two thirds of the stomach (defined as LSGC) and the remaining 430 patients with all other types of gastric cancer (OGC), and then clinicopathologically compared. Results showed that LP had deeper invasion $(\mathrm{p}=0.006)$, more frequent peritoneal dissemination including positive cytology $(\mathrm{p}=0.01$ and $\mathrm{p}=0.018)$ and lower curability $(\mathrm{p}=0.03$ ) compared with LSGC, whereas LSGC showed a higher malignant potential in a number of clinicopathological factors compared with OGC. Univariate analysis showed that survival in patients with LP was significantly poorer than in those with LSGC $(p=0.002)$ whose survival was, in turn, inferior to those with OGC. By contrast, LSGC was not a prognostic factor in SGC according to the multivariate analysis. The findings of this study suggested that the malignant status of LSGC differs from that of LP, and that curative gastrectomy is effective in improving the outcome for LSGC but not for LP, as LSGC may represent the prelinitis condition.
\end{abstract}

\section{Introduction}

Type IV scirrhous gastric cancer (SGC) is characterized by diffuse infiltration and proliferation of poorly differentiated

Correspondence to: Dr Kazuya Endo, Department of Surgery, Center for Gastroenterology, National Kyushu Medical Center, 1-8-1 Jigyohama, Fukuoka 810-8563, Japan

E-mail: k-endo@kyumed.jp

Key words: gastric cancer, scirrhous, linitis plastica, prognosis gastric cancer cells accompanied by marked stromal fibrosis (1). Linitis plastica (LP), also known as leather bottle stomach, is believed to be a typical case of SGC, usually involving the whole stomach and characterized by a grossly thickened wall. Despite recent advances in the diagnosis and treatment of gastric cancer, the majority of cases of SGC are not detected at an early stage since tumor cells migrate throughout the submucosa without severely affecting the mucosal lining of the stomach. This causes difficulty in detecting cancer cells by gastrointestinal series or endoscopy (2-5).

In LP, the cancer is poorly differentiated and originates in the gastric fundic gland area as a small IIc lesion, which shows extensive submucosal invasion without obvious concavities or recesses and a leather bottle-like appearance with giant folded walls. These walls are occasionally accompanied by peritoneal dissemination, considerable lymph node metastasis and direct invasion into the surrounding organs (6-8). Therefore, LP is recognized as a far-advanced gastric cancer in most cases, or as an early cancer that is accidentally detected in that it is difficult to diagnose the disease during progression from a small early stage lesion to leather bottle stomach at a stage known as the prelinitis condition (9).

Only a few studies regarding the prelinitis condition are available $(5,7,9)$. By contrast, numerous studies have commented on the definition (1-4), incidence (10), significance (11), pathology (12), prognosis (10-12) and management strategy (13-16) of SGC pertaining solely to the early and the final phase of the disease.

Although the prelinitis condition lacks an established definition, it can be clinically identified as type IV SGCs, other than LP, which do not involve the whole stomach, with lesions localized in part of the stomach, which are sometimes referred to as localized or partial SGC. Localized SGC is believed to be better than LP in terms of surgical curability and postsurgical prognosis, but a definitive report has not yet been provided.

The present study aimed to investigate the biological significance of localized SGC, to elucidate the progression of SGC to LP and to determine whether localized SGC has a prelinitis condition.

\section{Patients and methods}

Patients. A total of 509 consecutive patients with primary advanced gastric cancer who had undergone gastrectomy at the Department of Surgery, Center of Gastroenterology, National 
Kyushu Medical Center (Japan) between January 1994 and December 2004 were included in this study. The patients were divided into three groups comprising 19 patients with type IV scirrhous lesions invading the whole stomach (LP), 60 patients with type IV scirrhous lesions localized in less than two thirds of the stomach (LSGC) and the remaining 430 patients with all other types of gastric cancer (OGC).

Total gastrectomy was performed for 205 patients and distal or proximal gastrectomy for the remaining 304 patients; all resection margins had a macroscopically normal appearance. Cases with a macroscopic positive stump, due to obvious invasion of the esophagus and duodenum, were excluded from this study.

SGC was diagnosed by the macroscopic appearance of the surgical specimen and postoperative histological examination. The study population comprised 340 males and 169 females, whose ages ranged from 23 to 92 years, with a mean age of 66 . The patients were followed up, and only those who succumbed to gastric cancer were regarded as having succumbed to tumorrelated causes. The follow-up interval after surgery ranged from 2 days to 10 years and 11 months, with a mean interval of 4 years and 2 months. Clinicopathological results were assessed according to the general rules for clinical and pathological studies on gastric cancers based on the Japanese classification of gastric carcinoma (17) and tumor-node-metastasis staging criteria (18). In particular, the curative potential of gastric resection was adopted for its curability in the present study (17).

Definition of localized scirrhous gastric cancer. As mentioned previously, 79 SGC patients were divided into two groups of 19 patients with LP and 60 patients with LSGC. The stomach was anatomically divided into three sections: upper (U), middle (M) and lower (L) (Fig. 1) (17). Cases with lesions that invaded the duodenum or esophagus and those with a macroscopically positive stump were excluded. First, we reviewed the cases of 84 SGC patients who underwent gastrectomy, 5 of whom were excluded, leaving a total of 79 patients who were included in this study. Of the 79 SGC patients, total gastrectomy was performed in 51 and distal gastrectomy in the remaining 28 patients. The study population consisted of 50 males and 29 females, whose ages ranged from 29 to 87 years with a mean age of 65 . The follow-up interval after surgery ranged from 23 days to 10 years and 1 month with a mean interval of 2 years and 5 months.

Statistical analysis. The clinicopathological characteristics of the three groups were compared using the Chi-square test and Student's t-test. Cumulative survival rates were evaluated by the Kaplan-Meier method and the survival curves were tested by the Mantel-Cox method. Multivariate survival analysis was performed according to Cox's proportional hazards model in a forward stepwise manner. $\mathrm{P}<0.05$ was considered to be statistically significant.

\section{Results}

Comparison of clinicopathological findings among $O G C$, $L S G C$ and $L P$. Of the 509 patients with advanced gastric cancer included in the study, 60 (11.8\%) were classified as LSGC, $19(3.7 \%)$ as LP and the remaining $430(84.5 \%)$ as OGC. The
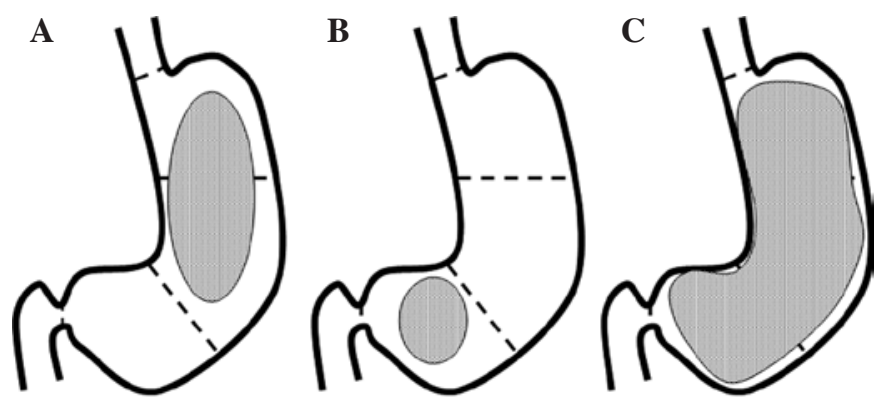

Figure 1. LSGC and LP are shown. (A) and (B) show LSGC with an invading lesion in less than two thirds of the stomach after the stomach was sectioned into three parts (U, M and L). (C) shows LP with an invading lesion of the entire stomach. LSGC, localized scirrhous gastric cancer; LP, linitis plastica; U, upper; M, middle; L, lower.

relationship between LP, LSGC and the clinicopathological characteristics of the patients are shown in Table I.

The LP group showed lower curability $(\mathrm{p}=0.03)$, higher mean age $(p=0.04)$ and deeper invasion to the gastric wall $(\mathrm{p}=0.006)$ compared with the LSGC group. Peritoneal dissemination and positive signs of peritoneal cytology (CY) were frequent in the LP group. No significant differences were noted in the distribution of gender, histological differentiation, frequency of venous and lymphatic invasion and in the incidence of lymph node metastasis. The relationship between LSGC and OGC is shown in Table II.

Significant differences were observed between the LSGC and OGC groups with regard to tumor size, stage, venous involvement, curability, depth of invasion, histological differentiation and peritoneal dissemination. The LSGC group showed significantly larger tumor size $(\mathrm{p}<0.0001)$, more advanced tumor stage $(\mathrm{p}=0.005)$, lower curability $(\mathrm{p}<0.0001)$, deeper invasion into the gastric wall $(\mathrm{p}<0.0001)$ and poorer histological differentiation $(\mathrm{p}<0.0001)$ compared with the OGC group. Peritoneal dissemination and venous involvement were more frequent in the LSGC group $(\mathrm{p}<0.0001$ and $\mathrm{p}<0.05)$ than in the OGC group. Severe lymphatic invasion was frequent in the LSGC group $(\mathrm{p}=0.002)$ (data not shown). Therefore, these factors suggest that LSGC is associated with more advanced and unfavorable clinicopathological findings compared with OGC but fewer compared with LP.

Survival comparison between OGC, LSGC and LP. The 1-, 3and 5-year survival rates of patients with OGC were 81.7, 61.1 and $53.8 \%$, respectively, which were significantly higher than those of patients with LSGC (73.2,36.5 and 29.8\%, respectively; $\mathrm{p}=0.0015$ ) (Fig. 2). However, the 1-, 3- and 5-year survival rates of patients with LP were 26.3, 21.1 and 7\%, respectively, which were significantly lower than those of LSGC $(\mathrm{p}=0.002)$ (Fig. 2). Of the 19 patients with LP, only 2 patients survived $>3$ years and only 1 patient survived $>5$ years.

Prognostic factors in patients with SGC. Multivariate analysis revealed that $\mathrm{CY}$ and curability were independently associated prognostic factors in patients with SGC (Table III), whereas SGC, lymph node metastasis, curability and TNM stage were independently associated prognostic factors in patients with advanced gastric cancer (Table IV). 
Table I. Clinicopathological characteristics of LSGC and LP.

\begin{tabular}{|c|c|c|c|}
\hline Variables & $\operatorname{LSGC}(n=60)$ & $\mathrm{LP}(\mathrm{n}=19)$ & P-value \\
\hline Gender & & & N.S. \\
\hline Male & $39(65 \%)$ & $11(57.9 \%)$ & \\
\hline Female & $21(35 \%)$ & $8(42.1 \%)$ & \\
\hline Age (years) & $63.1 \pm 12.5$ & $69.4 \pm 7.7$ & 0.04 \\
\hline Histology & & & N.S. \\
\hline Tub1 & $2(3.3 \%)$ & $0(0 \%)$ & \\
\hline Tub2 & $3(5 \%)$ & $4(21.1 \%)$ & \\
\hline Por1 & $9(15 \%)$ & $1(5.2 \%)$ & \\
\hline Por2 & $37(61.7 \%)$ & $11(57.9 \%)$ & \\
\hline Signet & $9(15 \%)$ & $3(15.8 \%)$ & \\
\hline Tumor depth & & & 0.006 \\
\hline $\mathrm{T} 2$ & $20(33.3 \%)$ & $2(10.5 \%)$ & \\
\hline $\mathrm{T} 3$ & $39(65 \%)$ & $13(68.4 \%)$ & \\
\hline $\mathrm{T} 4$ & $1(1.7 \%)$ & $4(21.1 \%)$ & \\
\hline Lymph node metastasis & & & N.S. \\
\hline Negative & $15(25 \%)$ & $2(10.5 \%)$ & \\
\hline Positive & $45(75 \%)$ & $17(89.5 \%)$ & \\
\hline Lymphatic invasion & & & N.S. \\
\hline Negative & $1(1.7 \%)$ & $0(0 \%)$ & \\
\hline Positive & $59(98.3 \%)$ & $19(100 \%)$ & \\
\hline Venous invasion & & & N.S. \\
\hline Negative & $19(31.6 \%)$ & $3(15.8 \%)$ & \\
\hline Positive & $41(68.4 \%)$ & $16(84.2 \%)$ & \\
\hline Peritoneal dissemination & & & 0.01 \\
\hline Negative & $50(83.3 \%)$ & $10(52.6 \%)$ & \\
\hline Positive & $10(16.7 \%)$ & $9(47.4 \%)$ & \\
\hline Peritoneal cytology & & & 0.018 \\
\hline Negative & $47(78.3 \%)$ & $9(47.4 \%)$ & \\
\hline Positive & $13(21.7 \%)$ & $10(52.6 \%)$ & \\
\hline Curability & & & 0.03 \\
\hline $\mathrm{A}, \mathrm{B}$ & $40(66.7 \%)$ & $7(36.8 \%)$ & \\
\hline $\mathrm{C}$ & $20(23.3 \%)$ & $12(63.2 \%)$ & \\
\hline TNM stage & & & N.S. \\
\hline $\mathrm{I}$ & $9(15 \%)$ & $1(5.3 \%)$ & \\
\hline II & $9(15 \%)$ & $1(5.3 \%)$ & \\
\hline III & $36(60 \%)$ & $11(57.8 \%)$ & \\
\hline IV & $6(10 \%)$ & $6(31.6 \%)$ & \\
\hline
\end{tabular}

N.S., not singnificant; Tub1, well-differentiated adenocarcinoma; Tub2, moderately differentiated adenocarcinoma; Por1, poorly differentiated adenocarcinoma, solid type; Por2, poorly differentiated adenocarcinoma, non-solid type; Signet, signet ring cell carcinoma; LP, linitis plastica; LSGC, localized type IV scirrhous gastric cancer; curability, curative potential of gastric resection (17).

\section{Discussion}

The classification of advanced gastric cancer into type I through IV by Borrmann has been globally accepted (19). The definition of SGC in this study is the same as that of Borrmann type IV and includes a number of grades of disease from LP to scirrhous pattern carcinoma. When the entire stomach wall is invaded by carcinoma cells, the stomach has a leather bottle-like appearance and the disease condition is known as LP, which is the typical and complete case of SGC. However, not all SGCs are defined as LP with a typical appearance. When only a section of the stomach is invaded by scirrhous pattern carcinoma cells, the case is also defined as SGC, although the stomach does not have a leather bottle-like appearance. Such atypical and localized cases of SGC (LSGC) have been classified in the same category as LP. However, the 
Table II. Clinicopathological characteristics of OGC and LSGC.

\begin{tabular}{|c|c|c|c|}
\hline Variables & $\operatorname{LSGC}(n=60)$ & OGC $(n=430)$ & P-value \\
\hline Gender & & & N.S. \\
\hline Male & $39(65 \%)$ & $290(67.4 \%)$ & \\
\hline Female & $21(35 \%)$ & $140(32.6 \%)$ & \\
\hline Age (years) & $63.1 \pm 12.5$ & $66.4 \pm 11.5$ & 0.04 \\
\hline Tumor size (mm) & $78.9 \pm 34.8$ & $56.4 \pm 29.6$ & $<0.0001$ \\
\hline Histology & & & $<0.0001$ \\
\hline Tub1, Tub2 & $5(8.3 \%)$ & $198(46.0 \%)$ & \\
\hline Por1, Por2 & $46(76.7 \%)$ & $169(39.4 \%)$ & \\
\hline Signet & $9(15 \%)$ & $41(9.5 \%)$ & \\
\hline Others & $0(0 \%)$ & $22(5.1 \%)$ & \\
\hline Tumor depth & & & $<0.0001$ \\
\hline $\mathrm{T} 2$ & $20(33.3 \%)$ & $258(60.0 \%)$ & \\
\hline $\mathrm{T} 3$ & $39(65 \%)$ & $155(36.0 \%)$ & \\
\hline $\mathrm{T} 4$ & $1(1.7 \%)$ & $17(4.0 \%)$ & \\
\hline Lymph node metastasis & & & N.S. \\
\hline Negative & $15(25 \%)$ & $145(33.7 \%)$ & \\
\hline Positive & $45(75 \%)$ & $285(66.3 \%)$ & \\
\hline Lymphatic invasion & & & N.S. \\
\hline Negative & $1(1.7 \%)$ & $34(7.9 \%)$ & \\
\hline Positive & $59(98.3 \%)$ & $396(92.1 \%)$ & \\
\hline Venous invasion & & & 0.048 \\
\hline Negative & $19(31.6 \%)$ & $197(45.8 \%)$ & \\
\hline Positive & $41(68.4 \%)$ & $233(54.2 \%)$ & \\
\hline Peritoneal dissemination & & & 0.0006 \\
\hline Negative & $50(83.3 \%)$ & $413(96.0 \%)$ & \\
\hline Positive & $10(16.7 \%)$ & $17(4.0 \%)$ & \\
\hline Liver metastasis & & & N.S. \\
\hline Negative & $59(98.3 \%)$ & $414(96.3 \%)$ & \\
\hline Positive & $1(1.7 \%)$ & $16(3.7 \%)$ & \\
\hline Curability & & & $<0.0001$ \\
\hline A, B & $40(66.7 \%)$ & $380(88.4 \%)$ & \\
\hline $\mathrm{C}$ & $20(23.3 \%)$ & $50(11.6 \%)$ & \\
\hline TNM stage & & & 0.005 \\
\hline $\mathrm{I}$ & $9(15 \%)$ & $118(27.5 \%)$ & \\
\hline II & $9(15 \%)$ & $108(25.1 \%)$ & \\
\hline III & $36(60 \%)$ & $158(36.7 \%)$ & \\
\hline IV & $6(10 \%)$ & $46(10.7 \%)$ & \\
\hline
\end{tabular}

N.S., not singnificant; Tub1, well-differentiated adenocarcinoma; Tub2, moderately differentiated adenocarcinoma; Por1, poorly differentiated adenocarcinoma, solid type; Por2, poorly differentiated adenocarcinoma, non-solid type; Signet, signet ring cell carcinoma; TNM, tumor metastases node; OGC, other types of advanced gastric cancer; LSGC, localized type IV scirrhous gastric cancer; curability, curative potential of gastric resection (17).

differences between LP and LSGC have not been studied. In the present study, we investigated the differences in malignant behavior between LP and LSGC using several clinical factors.

Our data demonstrated that LP is more aggressive in terms of peritoneal dissemination, depth of invasion, curability and peritoneal cytology than LSGC, and that the 5-year survival rate of patients with LSGC was significantly better than that of patients with LP.

Only $36.8 \%$ (7/19) of patients with LP underwent resection A or B compared with $66.7 \%$ (40/60) of patients with LSGC. Of the 19 patients with LP, 16 suffered from peritoneal recurrence and only one patient with LP survived for more than 
Table III. Factors independently associated with prognosis in SGC.

\begin{tabular}{lcccc}
\hline Variables & Standard error & Odds ratio & CI 95\% & P-value \\
\hline Peritoneal cytology & 0.456 & 2.207 & $0.150-0.894$ & 0.027 \\
LSGC (LP/LSGC) & 0.312 & 1.103 & $0.385-1.306$ & 0.269 \\
Serosal invasion & 0.385 & 0.900 & $0.333-1.504$ & 0.368 \\
Curability (A, B/C) & 0.431 & 2.885 & $0.124-0.671$ & 0.004 \\
Lymph node metastasis & 0.460 & 1.900 & $0.169-1.028$ & 0.057
\end{tabular}

CI, confidence interval; SGC, type IV scirrhous gastric cancer; LSGC, localized SGC; LP, linitis plastica; curability, curative potential of gastric resection (17).

Table IV. Factors independently associated with prognosis in advanced gastric cancer.

\begin{tabular}{|c|c|c|c|c|}
\hline Variables & Standard error & Odds ratio & CI $95 \%$ & P-value \\
\hline SGC (SGC/OGC) & 0.156 & 2.242 & $0.520-0.957$ & 0.025 \\
\hline Lymph node metastasis & 0.212 & 3.669 & $0.302-0.696$ & 0.0002 \\
\hline Curability (A, B/C) & 0.150 & 9.095 & $0.190-0.343$ & $<0.0001$ \\
\hline Stage (I, II/III, IV) & 0.173 & 2.994 & $0.425-0.836$ & 0.003 \\
\hline
\end{tabular}

CI, confidence interval; SGC, type IV scirrhous gastric cancer; OGC, other types of advanced gastric cancer; curability, curative potential of gastric resection (17).

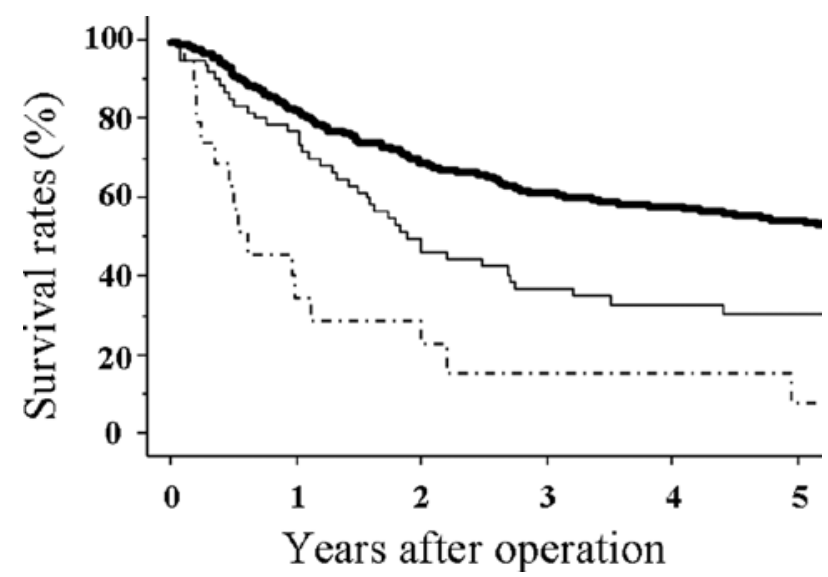

Figure 2. Survival curve of advanced gastric cancer including LSGC and LP. The prognosis of patients with LSGC (solid thin line) was significantly worse than that of OGC patients (solid thick line) $(\mathrm{p}=0.0015)$, whereas it was significantly better than that of LP patients (broken line) $(\mathrm{p}=0.002)$. LSGC, localized type IV scirrhous gastric cancer; LP, linitis plastica; OGC, other types of advanced gastric cancer.

5 years following surgery. Lymph node metastasis and venous invasion were frequent and TNM staging was more advanced in LP compared with LSGC, although no significant differences were observed.

These data suggest that LP is definitely a more advanced stage cancer with higher malignant potential than LSGC, and that surgical treatment for LP may not be effective in improving long-term survival. However, LSGC was also not easily treated. The data presented in this study also indicate that LSGC was aggressive in a number of prognostic factors, and that the 5-year survival rate of patients with LSGC was significantly worse than that of patients with OGC. SGC was an independent prognostic factor in advanced gastric cancer, as indicated by several previous studies (20-23). The malignant grade of LSGC is intermediate between OGC and LP.

SGC has been shown to possess unique clinicopathological characteristics and a poor survival outcome despite curative gastrectomy $(10-16,20-23)$. One reason for the poor prognosis specific to SGC is the difficulty in early detection. Poorly differentiated adenocarcinoma, developing from the fundic gland mucosa around the greater curvature of the stomach, are known to infiltrate submucosal tissue prior to ulceration of the primary lesion. This infiltration represents the initial lesions of LP, which are difficult to detect even by modern endoscopic examination (24). Furthermore, Oguro reported that the development of SGC was divided into both stable and rapid phases (7). SGC undergoes almost no change in the stable phase and then suddenly moves into the rapid phase. In the rapid phase, the development of SGC is extremely fast and is detected with difficulty, and early cancer or LP is usually identified as the disease condition of $\operatorname{SGC}(6,7)$, rather than the prelinitis condition. Certain studies have elucidated the mechanisms underlying the development of SGC and identified the prelinitis condition $(5,9)$. However, the definition of the prelinitis condition has yet to be established. In this study, LSGC may correspond to the prelinitis condition of the disease.

A new therapeutic strategy for SGC has been considered in order to improve its poor prognosis and determine the mechanisms and clinical course of SGC development. Clinical studies have demonstrated that preoperative chemotherapy, close staging by peritoneal cytology with exploratory laparoscopy, 
and strong chemotherapy, including S-1, may be a more favorable option than aggressive surgery or palliative gastrectomy $(2,3,13,16)$.

In the present study, peritoneal cytology was an independent prognostic factor in SGC. LP versus LSGC was not a prognostic factor, but surgical treatment for LP was not effective in prolonging patient survival, whereas a longer survival rate was expected in patients with LSGC who underwent curative resection. A consistent systematic chemotherapy was not performed for the 79 patients with SGC in this study, but the survival rate of patients who received chemotherapy was better than that of those who did not receive chemotherapy (data not shown), indicating that adequate systematic chemotherapy is essential for improving the outcome of SGC treatment.

In conclusion, we found that the clinicopathological characteristics and surgical outcomes of LSGC are different from those of LP and that its malignant grade is intermediate between OGC and LP. LP appears not to be a surgical disease, whereas long-term survival is expected in patients with LSGC who underwent curative resection. Furthermore, we believe that LSGC may represent the prelinitis condition.

\section{Acknowledgements}

The authors wish to express their gratitude to Drs S. Momosaki and Y. Nakayama of the Department of Pathology, National Kyushu Medical Center, Japan, for discussions related to pathology.

\section{References}

1. Takemura S, Yashiro M, Sunami T, et al: Novel models for human scirrhous gastric carcinoma in vivo. Cancer Sci 95: 893-900, 2004.

2. Ikeguchi M, Miyake T, Matsunaga T, et al: Recent results of therapy for scirrhous gastric cancer. Surg Today 39: 290-294, 2009.

3. Sasaki T, Koizumi W, Tanabe S, et al: TS-1 as first-line therapy for gastric linitis plastica: historical control study. Anticancer Drugs 17: 581-586, 2006.

4. Kodera Y, Nakanishi H, Ito S, et al: Detection of disseminated cancer cells in linitis plastica-type gastric carcinioma. Jpn J Clin Oncol 34: 525-531, 2004.

5. Morita K, Fujimori T, Ono Y, et al: Identification of the prelinitis condition in gastric cancer and analysis of TGF- $\beta$, TGF- $\beta$ RII and pS2 expression. Pathobiology 69: 321-328, 2001.

6. Matsukawa M, Kurihara M, Hirashima M, et al: Radiological findings of gastric scirrhous cancer. Gan To Kagaku Ryoho (in Japanese with English abstract) 21: 2378-2383, 1994.
7. Oguro Y: Endoscopic diagnosis of scirrhous gastric carcinoma. Gan To Kagaku Ryoho (in Japanese with English abstract) 21: 2384-2391, 1994.

8. Kohri Y, Takeda S and Kawai K: Earlier diagnosis of gastric infiltrating carcinoma (Scirrhous cancer). J Clin Gastroenterol 3: 17-20, 1981.

9. Ichikawa Y, Koshikawa N, Hasegawa S, et al: Marked increase of trypsin(ogen) in serum of linitis plastica (gastric cancer, Borrmann 4) patients. Clin Cancer Res 6: 1385-1388, 2000.

10. Kitamura K, Beppu R, Anai H, et al: Clinicopathological study of patients with Borrmann type IV gastric cancer. J Surg Oncol 58: 112-117, 1995.

11. An JY, Kang TH, Choi MG, et al: Borrmann Type IV: An independent prognostic factor for survival in gastric cancer. J Gastrointest Surg 12: 1364-1369, 2008.

12. Naito Y and Kino I: Pathogenesis and progression of scirrhous carcinoma. Gan To Kagaku Ryoho (in Japanese with English abstract) 21: 2364-2370, 1994.

13. Ikeguchi M, Yamamoto $\mathrm{O}$ and Kaibara N: Management protocol for scirrhous gastric cancer. In Vivo 18: 577-580, 2004.

14. Otsuji E, Kuriu Y, Okamoto K, et al: Outcome of surgical treatment for patients with scirrhous carcinoma of the stomach. Am J Surg 188: 327-332, 2004.

15. Kunisaki C, Shimada H, Nomura M, et al: Therapeutic strategy of scirrhous type gastric cancer. Hepatogastroenterology 52: 314-318, 2005.

16. Ikeguchi M, Matsumoto S, Yoshioka S, et al: Laparoscopic assisted intraperitoneal chemotherapy for patients with scirrhous gastric cancer. Chemotherapy 51: 15-20, 2005.

17. Japanese Gastric Cancer Association: Japanese Classification of Gastric Carcinoma - 2nd English edition. Gastric Cancer 1: 10-24, 1998.

18. Sobin LH, Gospodarowicz MK and Wittekind C: TNM Classification of Malignant Tumors. 7th edition. A John Wiley \& Sons, Ltd., NY, pp1-310, 2009.

19. Borrmann R: Geschwulstic des Magen und des Deudenums. In: Henke F, Lubarsch O (eds). Handbuch der Speziellen Pathologischen Anatomic und Histologie. vol IV, part 1. Springer Berlin, pp812-1054, 1926.

20. Nakamura R, Saikawa Y, Wada N, et al: Retrospective analysis of prognosis for scirrhous-type gastric cancer: one institution's experience. Int J Clin Oncol 12: 291-294, 2007.

21. Kodera Y, Yamamura Y, Ito S, et al: Is Borrmann type IV gastric carcinoma a surgical disease? An old problem revisited with reference to the result of peritoneal washing cytology. J Surg Oncol 78: 175-182, 2001.

22. Li C, Oh SJ, Kim S, et al: Macroscopic Borrmann type as a simple prognostic indicator in patients with advanced gastric cancer. Oncology 77: 197-204, 2009.

23. Chen CY, Wu CW, Lo SS, et al: Peritoneal carcinomatosis and lymph node metastasis are prognostic indicators in patients with Borrmann type IV gastric carcinoma. Hepatogastroenterology 49: 874-877, 2002.

24. Park MS, Ha HK, Choi BS, et al: Scirrhous gastric carcinoma: endoscopy versus upper gastrointestinal radiolography. Radiology 231: 421-426, 2004. 